\title{
THE EVANS-GRIFFITH SYZYGY THEOREM AND BASS NUMBERS
}

\author{
WINFRIED BRUNS
}

(Communicated by Louis J. Ratliff, Jr.)

\begin{abstract}
Let $(R, \mathfrak{m})$ be a Noetherian local ring containing a field. The syzygy theorem of Evans and Griffith (see The syzygy problem, Ann. of Math. (2) 114 (1981), 323-353) says that a nonfree $m$ th syzygy module $M$ over $R$ which has finite projective dimension must have rank $\geq m$. This theorem is an assertion about the ranks of the homomorphisms in certain acyclic complexes. It is the aim of this paper to demonstrate that the condition of acyclicity can be relaxed in a natural way. We shall use the generalization thus obtained to show that the Bass numbers of a module satisfy restrictions analogous to those which the syzygy theorem imposes on Betti numbers.
\end{abstract}

The acyclicity criterion of Buchsbaum and Eisenbud [3] is an essential tool in what follows. Theorem 1 below is the general version given by Northcott [14] which uses the polynomial or true grade of a module $M$ with respect to an ideal $I$ (see [14]); it is denoted by $\operatorname{Grade}(I, M)$. It is unnecessary for our considerations to know the exact definition of $\operatorname{Grade}(I, M)$. We will only need the inequality $\operatorname{Grade}(I, M) \geq \operatorname{grade}(I, M)$ where $\operatorname{grade}(I, M)$ denotes the "classical" grade of an ideal $I$ with respect to a module $M$; furthermore one should note that $\operatorname{Grade}(I, M)=\operatorname{grade}(I, M)$ if $M$ is a finite (i.e., finitely generated) module over a Noetherian ring $R$ [14]. Other notation to be explained: if $\varphi: F \rightarrow G$ is a homomorphism of finite free $R$-modules, then $I_{u}(\varphi)$ denotes the ideal generated by the $u$-minors of (a matrix) of $\varphi$. Furthermore, $\operatorname{rank}(\varphi, M)$ is the largest integer $v$ for which $I_{v}(\varphi) M \neq 0$. Several times we shall use that for a prime ideal $\mathfrak{p}$ one has $I_{u}(\varphi) \not \subset \mathfrak{p}$ if and only if $\operatorname{Im}\left(\varphi \otimes R_{\mathfrak{p}}\right)$ contains a free direct $R_{\mathfrak{p}}$-summand of rank $u$ of $G_{\mathfrak{p}}$; this is an easy consequence of Nakayama's lemma.

Theorem 1. Let $R$ be an arbitrary commutative ring,

$$
F_{0}: 0 \rightarrow F_{s} \stackrel{\varphi_{s}}{\rightarrow} F_{s-1} \rightarrow \cdots \rightarrow F_{1} \stackrel{\varphi_{1}}{\rightarrow} F_{0}
$$

a complex of finite free $R$-modules, and $M \neq 0$ an $R$-module. For $i=1, \ldots, s$ we set $r_{i}=\sum_{j=i}^{s}(-1)^{j-i} \operatorname{rank} F_{j}$.

(a) If $F . \otimes M$ is acyclic, then (i) $\operatorname{rank}\left(\varphi_{i}, M\right)=r_{i}$ for $i=1, \ldots, s$, and (ii) $\operatorname{Grade}\left(I_{r_{i}}\left(\varphi_{i}\right), M\right) \geq i$ for $i=1, \ldots, s$.

(b) Conversely, if condition (a)(ii) is satisfied, then $F . \otimes M$ is acyclic.

Received by the editors November 1, 1990 and, in revised form, January 28, 1991. 1980 Mathematics Subject Classification (1985 Revision). Primary 13C05, 13D25. 
Part (a) is [14, Theorem 14, p. 193] in a slightly different formulation. Part (b) is [14, Theorem 2, p. 248], except that we have omitted condition (a)(i) from its hypothesis; it is not difficult to derive from [14, Theorem 2, p. 101] that (a)(ii) implies (a)(i).

Our rings $R$ are always Noetherian and local, but the module $M$ appearing in the acyclicity criterion may be a balanced big Cohen-Macaulay module, i.e., an $R$-module $M$ such that every system of parameters of $R$ is an $M$-sequence. That such modules exist for local rings containing a field has been shown by Hochster [10]; see also Griffith [8] or Bartijn and Strooker [1]. The name "balanced" has been introduced by Sharp [17]; Sharp proves that the set of associated prime ideals of $M /\left(a_{1}, \ldots, a_{r}\right)$ is finite if $a_{1}, \ldots, a_{r}$ is an $M$-sequence. It is an easy exercise to verify that this property implies $\operatorname{grade}(I, M)=\operatorname{Grade}(I, M)$ for all ideals $I$ of $R$.

Let $(R, \mathfrak{m}, k)$ be a local ring. It is not a severe restriction to assume that a complex

$$
F:: 0 \rightarrow F_{s} \stackrel{\varphi_{s}}{\rightarrow} F_{s-1} \rightarrow \cdots \rightarrow F_{1} \stackrel{\varphi_{1}}{\rightarrow} F_{0}
$$

of finite free $R$-modules satisfies the conditions (i) $F_{s} \neq 0$ and (ii) $\varphi_{i}\left(F_{i}\right) \subset$ $\mathrm{m} F_{i-1}$ for $i=1, \ldots, s$. This is obvious for (i), and for (ii) one observes that $\varphi_{i}$ can be decomposed as $\varphi_{i}^{\prime} \oplus$ id: $F_{i}^{\prime} \oplus G \rightarrow F_{i-1}^{\prime} \oplus G$ with finite free $F_{i}^{\prime}, F_{i-1}^{\prime}$, and $G \neq 0$ if $\varphi_{i}\left(F_{i}\right) \not \subset \mathfrak{m} F_{i-1}$. Note that a minimal free resolution of a finite module over a Noetherian local ring satisfies these conditions automatically.

Our first result is that the ranks $r_{i}$ in the acyclicity criterion must be positive for the complexes just considered.

Proposition 1. Let $(R, \mathfrak{m}, k)$ be a local ring, and

$$
F .: 0 \rightarrow F_{s} \stackrel{\varphi_{s}}{\rightarrow} F_{s-1} \rightarrow \cdots \rightarrow F_{1} \stackrel{\varphi_{1}}{\rightarrow} F_{0}
$$

a complex of finite free $R$-modules with $F_{s} \neq 0$ and $\varphi_{i}\left(F_{i}\right) \subset \mathfrak{m} F_{i-1}$ for $i=$ $1, \ldots, s$. Suppose there exists an $R$-module $M$ such that $M \neq \mathfrak{m} M$ and $F . \otimes M$ is acyclic. As before, set $r_{i}=\sum_{j=1}^{s}(-1)^{j-i} \operatorname{rank} F_{j}$. Then $r_{i} \geq 1$ for $i=1, \ldots, s$.

Proof. One has $r_{s}=\operatorname{rank} F_{s} \geq 1$ by hypothesis, and it follows from Theorem 1 that $r_{i}=\operatorname{rank}\left(\varphi_{i}, M\right) \geq 0$ for all $i$. Arguing inductively, we have only to show: $r_{1}=0$ implies $r_{2}=0$.

If $r_{1}=\operatorname{rank}\left(\varphi_{1}, M\right)=0$, then obviously $\varphi_{1} \otimes M=0$. Therefore we have an exact sequence

$$
F_{2} \otimes M \stackrel{\varphi_{2} \otimes M}{\rightarrow} F_{1} \otimes M \rightarrow 0 .
$$

Consequently $F_{2} \otimes M \otimes k \rightarrow F_{1} \otimes M \otimes k \rightarrow 0$ is also exact. By hypothesis $M \neq \mathrm{m} M$, equivalently, $M \otimes k$ is a nonzero $k$-vector space. Thus the sequence

$$
F_{2} \otimes k \stackrel{\varphi_{2} \otimes k}{\rightarrow} F_{1} \otimes k \rightarrow 0
$$

of finite-dimensional $k$-vector spaces must be exact. On the other hand, $\varphi_{2} \otimes$ $k=0$ since $\varphi_{2}\left(F_{2}\right) \subset \mathfrak{m} F_{1}$. Hence we get $F_{2}=0$, and $r_{2}=\operatorname{rank} F_{2}-r_{1}=0$.

All the proofs of the syzygy theorem of Evans and Griffith use the notion of order ideal in an essential way. Let $M$ be a module over a commutative ring $R$, and $x \in M$. Then

$$
\mathscr{O}(x)=\left\{\alpha(x): \alpha \in \operatorname{Hom}_{R}(M, R)\right\}
$$


is called the order ideal of $x$. Suppose that $F$ is a free module with basis $e_{1}, \ldots, e_{n}$. For $x \in F$ with representation $x=a_{1} e_{1}+\cdots+a_{n} e_{n}$ one obviously has $\mathscr{O}(x)=\left(a_{1}, \ldots, a_{n}\right)$.

The following theorem and its proof are direct generalizations of EvansGriffith [6, Theorem 3.14 and its proof].

Theorem 2. Let $(R, \mathfrak{m})$ be a local ring containing a field. Let

$$
F .: 0 \rightarrow F_{s} \stackrel{\varphi_{s}}{\rightarrow} F_{s-1} \rightarrow \cdots \rightarrow F_{1} \stackrel{\varphi_{1}}{\rightarrow} F_{0}
$$

be a complex of finite free $R$-modules such that

$$
\operatorname{dim} R / I_{r_{i}}\left(\varphi_{i}\right) \leq \operatorname{dim} R-t-i, \quad i=1, \ldots, s,
$$

where $r_{i}=\sum_{j=i}^{s}(-1)^{i-j} \operatorname{rank} F_{j}$ and $t \geq 0$. Then, for $j=1, \ldots, s$ and every $e \in F_{j}$ with $e \notin \mathfrak{m} F_{j}+\operatorname{Im} \varphi_{j+1}$ one has $\operatorname{dim} R / \mathscr{O}\left(\varphi_{j}(e)\right) \leq \operatorname{dim} R-t-j$.

Proof. Adjusting $t$ and the indices, one may assume that $j=1$. Let $J=$ $\mathscr{O}\left(\varphi_{1}(e)\right)$. There is nothing to prove if $J=R$. So assume that $J \subset \mathrm{m}$.

We put $\bar{R}=R / J$ and $\bar{F}=F \cdot \otimes \bar{R}$. From the description of $J$ above one gets $\bar{\varphi}_{1}(\bar{e})=0$. In order to derive a contradiction, we assume $\operatorname{dim} R / J \geq \operatorname{dim} R-t$. Note that $I_{r_{i}}\left(\bar{\varphi}_{i}\right)=\left(I_{r_{i}}\left(\varphi_{i}\right)+J\right) / J$. Hence

$$
\operatorname{dim} \bar{R} / I_{r_{i}}\left(\bar{\varphi}_{i}\right) \leq \operatorname{dim} R / I_{r_{i}}\left(\varphi_{i}\right) \leq \operatorname{dim} R-i-t \leq \operatorname{dim} \bar{R}-i .
$$

Therefore $I_{r_{i}}\left(\bar{\varphi}_{i}\right)$ contains a sequence $x_{1}, \ldots, x_{i}$ which is part of a system of parameters for $\bar{R}$. It follows that $\operatorname{grade}\left(I_{r_{i}}\left(\bar{\varphi}_{i}\right), M\right) \geq i$ for a balanced big Cohen-Macaulay module $M$ of $\bar{R}$. By the acyclicity criterion $\bar{F} \cdot \otimes M$ is acyclic. Because of $\bar{\varphi}_{i}(\bar{e})=0$ we have $\left(\bar{\varphi}_{1} \otimes M\right)(\bar{e} \otimes M)=0$. Let $C=\operatorname{Coker} \varphi_{2}$, and $\pi: F_{1} \rightarrow C$ be the natural epimorphism. Since $\bar{F}^{\prime} . \otimes M$ is acyclic, $\bar{\varphi}_{2} \otimes M$ induces an isomorphism $\bar{C} \otimes M \rightarrow \operatorname{Im}\left(\bar{\varphi}_{1} \otimes M\right)$. So $\bar{\pi}(\bar{e}) \otimes M=0$.

On the other hand, the hypothesis $e \notin \mathfrak{m} F_{1}+\operatorname{Im} \varphi_{2}$ implies that $\bar{\pi}(\bar{e}) \notin$ $\mathfrak{m} \bar{C}$. Thus the image of $\bar{\pi}(\bar{e}) \otimes M$ under the natural epimorphism $\bar{C} \otimes M \rightarrow$ $(\bar{C} / \mathfrak{m} \bar{C}) \otimes(M / \mathfrak{m} M)$ is isomorphic with $M / \mathfrak{m} M \neq 0$, a contradiction.

In view of the methods of Bruns [2] it is only a technical exercise now to obtain the following generalization of the syzygy theorem. For $t=0$ the condition on the complex $F$. is related to the notion of phantom acyclicity introduced by Hochster and Huneke (see [11, Theorem 9.8]), and their methods easily yield an analogous result.

Theorem 3. Let $(R, \mathfrak{m}, k)$ be a Noetherian local ring containing a field. Consider a complex

$$
F .: 0 \rightarrow F_{s} \stackrel{\varphi_{s}}{\rightarrow} F_{s-1} \rightarrow \cdots \rightarrow F_{1} \stackrel{\varphi_{1}}{\rightarrow} F_{0}
$$

of finite free $R$-modules with $F_{s} \neq 0$ and $\varphi_{i}\left(F_{i}\right) \subset \mathfrak{m} F_{i-1}$ for $i=1, \ldots, s$. Suppose that

$$
\operatorname{dim} R / I_{r_{i}}\left(\varphi_{i}\right) \leq \operatorname{dim} R-t-i, \quad i=1, \ldots, s,
$$

where $r_{i}=\sum_{j=i}^{s}(-1)^{i-j} \operatorname{rank} F_{j}$ and $t \geq 0$. Then $r_{i} \geq t+i$ for $i=1, \ldots, s-1$. Proof. In order to show that $r_{i} \geq t+i$ we can truncate the complex at $F_{i-1}$, adjust the indices, and replace $t$ by $t+i-1$. Therefore it is enough to show that $r_{1} \geq t+1$. Note that there is nothing to prove if $s=1$. 
Let $M$ be a balanced big Cohen-Macaulay module for $R$. As in the proof of the previous theorem it follows that $F . \otimes M$ is acyclic. From Proposition 1 one gets that $r_{i} \geq 1$ for $i=1, \ldots, s$. This inequality covers the case $t=0$.

Let $t \geq 1$. Since $\operatorname{rank}\left(\varphi_{1}, M\right)=r_{1} \geq 1$, we have $\operatorname{rank} F_{1} \geq r_{1} \geq 1$. As $\varphi_{2}\left(F_{2}\right) \subset \mathfrak{m} F_{1}$, there exists $e \in F_{1}$ with $e \notin \mathfrak{m} F_{1}+\operatorname{Im} \varphi_{2}$. Put $F_{1}^{\prime}=F_{1} / R e$, and choose $\varphi_{2}^{\prime}$ as the induced map $F_{2} \rightarrow F_{1}^{\prime}$. Let $\mathfrak{p}$ be a prime ideal with $\operatorname{dim} R / \mathfrak{p} \geq \operatorname{dim} R-t$. Then $I_{r_{2}}\left(\varphi_{2}\right) \not \subset \mathfrak{p}$ and $I_{r_{1}}\left(\varphi_{1}\right) \not \subset \mathfrak{p}$. Since $r_{1}+r_{2}=$ $\operatorname{rank} F_{2}$, one sees easily that the sequence

$$
0 \rightarrow \operatorname{Im}\left(\varphi_{2} \otimes R_{\mathfrak{p}}\right) \rightarrow F_{2} \otimes R_{\mathfrak{p}} \rightarrow \operatorname{Im}\left(\varphi_{1} \otimes R_{\mathfrak{p}}\right) \rightarrow 0
$$

is split exact. By Theorem 2 we know that $\mathscr{O}\left(\varphi_{1}(e)\right) \not \subset \mathfrak{p}$; therefore $\varphi_{1}(e)$ generates a free direct summand of $\operatorname{Im}\left(\varphi_{1} \otimes R_{\mathfrak{p}}\right)$. Hence $\operatorname{Im}\left(\varphi_{2}^{\prime} \otimes R_{\mathfrak{p}}\right)$ is a free direct summand of rank $r_{2}$ of $F_{1}^{\prime}$. This implies $I_{r_{2}}\left(\varphi_{2}^{\prime}\right) \not \subset \mathfrak{p}$, whence $\operatorname{dim} R / I_{r_{2}^{\prime}}\left(\varphi_{2}^{\prime}\right) \leq \operatorname{dim} R-t-1$.

Set $C=\operatorname{Coker} \varphi_{2}$ and choose an epimorphism $\pi: G \rightarrow C^{*}$; as usual $C^{*}=$ $\operatorname{Hom}_{R}(C, R)$. Composing $\pi^{*}$ with the natural homomorphism $C \rightarrow C^{* *}$ one gets a map $\psi: C \rightarrow G^{*}$, and it is easily seen that for every prime ideal $\mathfrak{p}$ and every free direct summand $N$ of $C_{\mathfrak{p}}$ one has that $\left(\psi \otimes R_{\mathfrak{p}}\right)(N)$ is a free direct $R_{\mathfrak{p}}$-summand of $G^{*} \otimes R_{\mathfrak{p}}$. So we take $F_{0}^{\prime}=G^{*}$ and choose $\varphi_{1}^{\prime}$ as the homomorphism $F_{1}^{\prime} \rightarrow F_{0}^{\prime}$ induced by $\psi$. If, as above, $\operatorname{dim} R / \mathfrak{p} \geq \operatorname{dim} R-t$, then $\operatorname{Im} \varphi_{1}^{\prime} \otimes R_{\mathfrak{p}}$ contains a free direct $R_{\mathfrak{p}}$-summand of $\left(F_{0}^{\prime}\right)_{\mathfrak{p}}$ of $\operatorname{rank} r_{1}^{\prime}=$ $r_{1}-1$. So we get $\operatorname{dim} R / I_{r_{1}^{\prime}}\left(\varphi_{1}^{\prime}\right) \leq \operatorname{dim} R-t-1$, too. Furthermore, as $s>$ $1, F_{s}$ has not been touched. Finally, one obviously has $\varphi_{2}^{\prime}\left(F_{2}\right) \subset \mathfrak{m} F_{1}^{\prime}$. If $\varphi_{1}^{\prime}\left(F_{1}\right) \not \subset \mathfrak{m} F_{0}^{\prime}$, then one can decompose $\varphi_{1}^{\prime}$ in the form $\varphi_{1}^{\prime \prime} \oplus$ id as discussed above Proposition 1; replacing $\varphi_{1}^{\prime}$ by $\varphi_{1}^{\prime \prime}$ does not change the situation in an essential way. Therefore an inductive argument applies to the complex

$$
F_{.}^{\prime}: 0 \rightarrow F_{s} \rightarrow F_{s-1} \rightarrow \cdots \rightarrow F_{2} \stackrel{\varphi_{2}^{\prime}}{\rightarrow} F_{1}^{\prime} \stackrel{\varphi_{1}^{\prime}}{\rightarrow} F_{0}^{\prime} .
$$

It seems that all the proofs of the syzygy theorem given by Evans and Griffith [4-6] require a weak condition on the underlying ring. Theorem 3 contains the syzygy theorem as stated at the beginning; in this generality it has also been proved by Ogoma [15].

Corollary 1. Let $R$ be a Noetherian local ring containing a field, and $M$ an $m$ th syzygy module of finite projective dimension. If $M$ is not free, then $\operatorname{rank} M \geq m$.

Proof. There is an exact sequence $F_{s}: 0 \rightarrow F_{s} \stackrel{\varphi_{s}}{\rightarrow} F_{s-1} \rightarrow \cdots \rightarrow F_{1} \stackrel{\varphi_{1}}{\rightarrow} F_{0}$ such that $M=\operatorname{Im} \varphi_{m}$, and furthermore $F_{s} \neq 0$ and $\varphi_{s}\left(F_{s}\right) \subset \mathfrak{m} F_{s-1}:$ simply splice an exact sequence in which $M$ appears as an $m$ th syzygy, with a minimal free resolution of $M$. The acyclicity criterion yields that $\operatorname{grade} I_{r_{i}}\left(\varphi_{i}\right) \geq i$. A fortiori one has $\operatorname{dim} R / I_{r_{i}}\left(\varphi_{i}\right) \leq \operatorname{dim} R-i$. Since $M$ is not free, it follows that $m<s$. So rank $M=r_{m} \geq m$ results directly from the theorem.

In view of Proposition 1 one may ask whether the condition on $\operatorname{dim} R / I_{r_{i}}\left(\varphi_{i}\right)$ in Theorem 3, for the case in which $t=0$, can be replaced by the requirement that there exists an $R$-module $M$ with $M \neq \mathrm{m} M$ for which $F . \otimes M$ is acyclic. The following corollary shows that this is possible if $M$ is finite. Another suitable condition is that the homology of $F$. has codimension $\geq s$ :

Corollary 2. Let $R$ be a Noetherian local ring containing a field, and

$$
F_{0}: 0 \rightarrow F_{s} \stackrel{\varphi_{s}}{\rightarrow} F_{s-1} \rightarrow \cdots \rightarrow F_{1} \stackrel{\varphi_{1}}{\rightarrow} F_{0}
$$


a complex of finite free $R$-modules with $F_{s} \neq 0$ and $\varphi_{i}\left(F_{i}\right) \subset \mathfrak{m} F_{i-1}$ for $i=$ $1, \ldots, s$. Suppose that one of the following conditions is satisfied:

(a) there exists a finite $R$-module $M \neq 0$ for which $F . \otimes M$ is acyclic;

(b) $\operatorname{dim} H_{i}\left(F_{0}\right) \leq \operatorname{dim} R-s$ for $i=1, \ldots, s$.

Then $r_{i} \geq i$ for $i=1, \ldots, s-1$.

Proof. (a) We may first replace $R$ and $F$. by $R /$ Ann $M$ and $F . \otimes R /$ Ann $M$ respectively, and thus assume $\operatorname{Supp} M=\operatorname{Spec} R$. The acyclicity criterion yields $\operatorname{grade}\left(I_{r_{i}}\left(\varphi_{i}\right), M\right)$

$\geq i$ for all $i$. Since $M$ is finite and $\operatorname{Supp} M=\operatorname{Spec} R$, it is easily seen that $\operatorname{dim} R / I_{r_{i}}\left(\varphi_{i}\right) \leq \operatorname{dim} R-i$. Therefore the hypotheses of the theorem are satisfied with $t=0$.

(b) Assume that $\operatorname{dim} R / I_{r_{i}}\left(\varphi_{i}\right)>\operatorname{dim} R-i$ for some $i$, and let $\mathfrak{p}$ be a prime ideal containing $I_{r_{i}}\left(\varphi_{i}\right)$ such that $\operatorname{dim} R / \mathfrak{p}=\operatorname{dim} R / I_{r_{i}}\left(\varphi_{i}\right)$. By hypothesis the complex $F_{\bullet} \otimes R_{\mathfrak{p}}$ is acyclic. Therefore the acyclicity criterion implies $\operatorname{grade}\left(I_{r_{i}}\left(\varphi_{i}\right)\right)_{\mathfrak{p}} \geq i$. A fortiori one has height $I_{r_{i}}\left(\varphi_{i}\right) \geq i$. This inequality contradicts the assumption that $\operatorname{dim} R / I_{r_{i}}\left(\varphi_{i}\right)>\operatorname{dim} R-i$.

We cannot present a counterexample to this corollary for nonfinite $M$. One should note, however, that the inequality used in its proof, namely, $\operatorname{grade}(I, M)$ $\leq \operatorname{dim} R-\operatorname{dim} R / I$ if $\operatorname{Supp} M=\operatorname{Spec} R$, does not hold in general, not even for a balanced big Cohen-Macaulay module $M$. One of Nagata's famous counterexamples is a three-dimensional noncatenary local domain $R$ containing a field.[13]. Thus $R$ has a balanced big Cohen-Macaulay module $M$. Sharp observed in [17] that $\operatorname{Supp} M=\operatorname{Spec} R$ and that the so-called little support supp $M$ of $M$ (denoted supersupp $M$ in [17]) is a proper subset of $\operatorname{Spec} R$. Then Theorem 3.6 of Zarzuela [18] implies that there exists a system of parameters $x_{1}, x_{2}, x_{3}$ for $M$ which is not a system of parameters for $R$, hence $\operatorname{dim} R /\left(x_{1}, x_{2}, x_{3}\right)>0$. On the other hand, $x_{1}, x_{2}, x_{3}$ is an $M$-sequence by [18, Theorem 3.3].

Let $R$ be a Noetherian ring and $M$ a finite $R$-module. The Bass numbers

$$
\mu_{i}(\mathfrak{p}, M)=\operatorname{dim}_{k(\mathfrak{p})} \operatorname{Ext}_{R_{\mathfrak{p}}}^{i}\left(k(\mathfrak{p}), M_{\mathfrak{p}}\right), \quad \mathfrak{p} \in \operatorname{Spec} R,
$$

determine the minimal injective resolution

$$
I^{\bullet}: 0 \rightarrow E^{0}(M) \rightarrow E^{1}(M) \rightarrow \cdots \rightarrow E^{i}(M) \rightarrow \cdots
$$

of $M$; it is well known that $E^{i}(M)=\bigoplus_{\mathfrak{p} \in \operatorname{Spec} R} E(R / \mathfrak{p})^{\mu_{i}(\mathfrak{p}, M)}$ for all $i \geq 0$. Here $E(R / \mathfrak{p})$ denotes the injective hull of $R / \mathfrak{p}$ and $k(\mathfrak{p})$ is the field $R_{\mathfrak{p}} / \mathfrak{p} R_{\mathfrak{p}}$. (Matsumura [12] contains all the results about injective modules needed below.) In the following we want to derive inequalities satisfied by the numbers $\mu_{i}(\mathfrak{m}, M)$ when $(R, \mathfrak{m}, k)$ is a local ring; since the Bass numbers are local data by definition, such inequalities can be translated into assertions about the $\mu_{i}(\mathfrak{p}, M)$ in general. It is easily seen that the Bass numbers $\mu_{i}(\mathfrak{m}, M)$ are invariant under completion. Therefore we may assume that $R$ is complete; then $\operatorname{End}(E(k))=R$, a crucial fact in what follows. For simplicity of notation we set $\mu_{i}=\mu_{i}(\mathfrak{m}, M)$.

The best inequalities so far have been given by Foxby [7]. As Foxby did, we use the idea of Peskine-Szpiro [16] which is to construct a complex of finite free $R$-modules whose ranks are the Bass numbers $\mu_{i}$. Let $\Gamma_{\mathfrak{m}}(-)$ denote the functor which assigns every module its submodule of elements annihilated by 
a power of $\mathfrak{m}$. Since every element in $E(R / \mathfrak{p})$ is annihilated by a power of $\mathfrak{p}$, an application of $\Gamma_{\mathfrak{m}}(-)$ to $I^{\bullet}$ yields the subcomplex

$$
J^{\cdot}: 0 \rightarrow E(k)^{\mu_{0}} \stackrel{\sigma_{0}}{\rightarrow} \cdots \stackrel{\sigma_{i-1}}{\rightarrow} E(k)^{\mu_{i}} \stackrel{\sigma_{i}}{\rightarrow} \cdots .
$$

The cohomology module $H^{i}\left(J^{\bullet}\right)$ is the $i$ th local cohomology $H_{\mathfrak{m}}^{i}(M)$ of $M$; see Grothendieck [9]. Since $\operatorname{End}(E(k))=R, \operatorname{Hom}_{R}\left(E(k), J^{\bullet}\right)$ is a complex

$$
G^{\cdot}=\operatorname{Hom}_{R}\left(E(k), J^{\cdot}\right): 0 \rightarrow R^{\mu_{0}} \stackrel{\psi_{0}}{\rightarrow} R^{\mu_{1}} \rightarrow \cdots \stackrel{\psi_{i-1}}{\rightarrow} R^{\mu_{i}} \stackrel{\psi_{i}}{\rightarrow} \cdots
$$

of finite free $R$-modules; furthermore the maps $\sigma_{i}$ can be considered matrices over $R$, and $\psi_{i}$ is given by the same matrix as $\sigma_{i}$. Since $I^{\cdot}$ is a minimal injective resolution, the entries of these matrices are in $\mathfrak{m}$.

Applying $\operatorname{Hom}_{R}(-, E(k))$ to $J^{\bullet}$ one obtains another complex of finite free $R$-modules:

$$
I .=\operatorname{Hom}_{R}\left(J^{\cdot}, E(k)\right): \cdots \stackrel{\chi_{i}}{\rightarrow} R^{\mu_{i}} \stackrel{\chi_{i-1}}{\rightarrow} \cdots \stackrel{\chi_{1}}{\rightarrow} R^{\mu_{1}} \stackrel{\chi_{0}}{\rightarrow} R^{\mu_{0}} \rightarrow 0 ;
$$

the matrix representing $\chi_{i}$ is obviously the transpose of $\sigma_{i}$. Let ${ }^{*}$ denote the functor $\operatorname{Hom}_{R}(-, R)$. As just seen,

$$
\left(G^{*}\right)^{*}=L . \text { and }\left(L_{\cdot}\right)^{*}=G^{*} \text {. }
$$

The advantage of $L$. over $G^{*}$ is that we know its homology. By the exactness of $\operatorname{Hom}_{R}(-, E(k))$ one has

$$
H_{i}\left(L_{\bullet}\right) \cong \operatorname{Hom}_{R}\left(H^{i}\left(J^{\bullet}\right), E(k)\right)=\operatorname{Hom}_{R}\left(H_{\mathfrak{m}}^{i}(M), E(k)\right) .
$$

Now it follows readily from a well-known vanishing theorem of local cohomology that $\operatorname{dim} H_{i}\left(L_{*}\right) \leq i$; see [7, Remark (2.7)] for the details.

In order to adapt the present notation to those of Theorem 3 we set $d=$ $\operatorname{dim} R, \nu_{i}=\mu_{d-i}, \varphi_{i}=\psi_{d-i}$, and define the complex $F$. by

$$
F .: 0 \rightarrow R^{\nu_{d}} \stackrel{\varphi_{d}}{\rightarrow} R^{\nu_{d-1}} \rightarrow \cdots \rightarrow R^{\nu_{1}} \stackrel{\varphi_{1}}{\rightarrow} R^{\nu_{0}} .
$$

We want to show that $F$. satisfies the condition $\operatorname{dim} R / I_{r_{i}}\left(\varphi_{i}\right) \leq d-i$ where $r_{i}=$ $\sum_{j=1}^{d}(-1)^{i-j} \nu_{j}$. Because of the duality between $F$. and $L$. one has $I_{r_{i}}\left(\varphi_{i}\right)=$ $I_{s_{d-i}}\left(\chi_{d-i}\right)$ with $s_{v}=\sum_{j=0}^{v}(-1)^{j} \mu_{j}$. Consider the truncation

$$
(L . \mid d-i+1): R^{\mu_{d-i+1}} \stackrel{\chi_{d-i}}{\rightarrow} R^{\mu_{d-i}} \rightarrow \cdots \rightarrow R^{\mu_{1}} \stackrel{\chi_{0}}{\rightarrow} R^{\mu_{0}} \rightarrow 0 .
$$

Since $\operatorname{dim} H_{v}\left(L_{\cdot}\right) \leq v$, the complex $(L \cdot \mid d-i+1) \otimes R_{\mathfrak{p}}$ is exact for prime ideals $\mathfrak{p}$ such that $\operatorname{dim} R / \mathfrak{p} \geq d-i+1$. Then $(L \cdot \mid d-i+1) \otimes R_{\mathfrak{p}}$ must even be split exact. This property carries over to $(L \cdot \mid d-i+1) \otimes R_{\mathfrak{p}} / \mathfrak{p} R_{\mathfrak{p}}$, and elementary linear algebra shows that $I_{s_{d-i}}\left(\chi_{d-i}\right) \not \subset \mathfrak{p}$. Altogether one concludes that $\operatorname{dim} R / I_{s_{d-i}}\left(\chi_{d-i}\right) \leq d-i$ as desired.

Let $t=\operatorname{depth} M$. Since $t=\min \left\{i: \operatorname{Ext}_{R}^{i}(k, M) \neq 0\right\}=\min \left\{i: \mu_{i} \neq 0\right\}$, one has $R^{\nu_{d-t+j}}=0$ for $j \geq 1$ and $R^{\nu_{d-t}} \neq 0$. Moreover, as noticed above, $\varphi_{i}\left(R^{\nu_{i}}\right) \subset \mathfrak{m} R^{\nu_{i-1}}$. Omitting the zero terms at the left-hand side of $F$. yields the complex

$$
0 \rightarrow R^{\nu_{d-t}} \stackrel{\varphi_{d-t}}{\rightarrow} R^{\nu_{d-t-1}} \rightarrow \cdots \rightarrow R^{\nu_{1}} \stackrel{\varphi_{1}}{\rightarrow} R^{\nu_{0}}
$$

which satisfies the hypotheses of Theorem 3 . Thus

$$
\mu_{d-i}=\nu_{i}=r_{i+1}+r_{i} \geq \begin{cases}1, & i=d-t \\ d-t, & i=d-t-1 \\ 2 i+1, & i=0, \ldots, d-t-2\end{cases}
$$


We state this result formally as a part (a) of the following theorem; part (b) is due to Foxby [7] and has been included for completeness.

Theorem 4. Let $R$ be a Noetherian local ring containing a field, $\operatorname{dim} R=d$, and $M$ a finite $R$-module of depth $t$.

(a) Then one has

$$
\mu_{i}(\mathfrak{m}, M) \geq \begin{cases}1, & i=t, \\ d-t, & i=t+1, \\ 2(d-i)+1, & i=t+2, \ldots, d .\end{cases}
$$

(b) If $t<\operatorname{dim} M=d$, then $\mu_{d}(\mathfrak{m}, M) \geq 2$.

Remarks. (a) If $R$ is a Cohen-Macaulay local ring (possibly of mixed characteristic), then $\operatorname{dim} R / I_{r_{i}}\left(\varphi_{i}\right) \leq \operatorname{dim} R-i$ implies that grade $I_{r_{i}}\left(\varphi_{i}\right) \geq i$. Therefore the complex $F$. defined above Theorem 4 is acyclic, and Proposition 1 already yields

$$
\mu_{i}(M, \mathfrak{m}) \geq \begin{cases}1, & i=\operatorname{depth} M \text { and } i=\operatorname{dim} R, \\ 2, & \text { depth } M<i<\operatorname{dim} R .\end{cases}
$$

This inequality has been obtained by Foxby [7] for Cohen-Macaulay local rings and local rings containing a field.

(b) Theorem 3 and its consequences admit conclusions for Noetherian local rings $(R, \mathfrak{m})$ which do not contain a field. Let $p=\operatorname{char} R / \mathfrak{m}$ and set $\bar{R}=$ $R /(p)$. If $F$. is a complex satisfying the hypotheses of Theorem 3 , then $F . \otimes \bar{R}$ again satisfies them after one has replaced $t$ by $t-1$. Since $\bar{R}$ contains a field, one obtains the inequalities $r_{i} \geq t+i-1$. Consequently these inequalities with $t=0$ hold in Corollary 2, too, and in Corollary 1 one must replace $m$ by $m-1$. The modification of Theorem $4(a)$ is left to the reader.

\section{REFERENCES}

1. J. Bartijn and J. R. Strooker, Modifications monomiales, Séminaire d'Algébre (P. Dubreil and M.-P. Malliavin, eds.), Lecture Notes in Math., vol. 1029, Springer-Verlag, New York, pp. $192-217$.

2. W. Bruns, 'Jede' endliche freie Auflösung ist freie Auflösung eines von drei Elementen erzeugten Ideals, J. Algebra 39 (1976), 429-439.

3. D. Buchsbaum and D. Eisenbud, What makes a complex exact, J. Algebra 25 (1973), 259268.

4. E. G. Evans and P. Griffith, The syzygy problem, Ann. of Math. (2) 114 (1981), 323-353.

5. __ The syzygy theorem: a new proof and historical perspective ( $R$. Y. Sharp, ed.), (Commutative Algebra: Durham 1981), London Math. Soc. Lecture Note Ser., vol. 72, Cambridge Univ. Press, New York, 1982, pp. 2-11.

6. __ Syzygies, London Math. Soc. Lecture Note Ser., vol. 106, Cambridge Univ. Press, Cambridge, 1985.

7. H.-B. Foxby, On the $\mu^{i}$ in a minimal injective resolution. II, Math. Scand. 41 (1977), 19-44.

8. P. Griffith, Maximal Cohen-Macaulay modules and representation theory, J. Pure Appl. Algebra 13 (1978), 321-334.

9. A. Grothendieck, Local Cohomology, Lecture Notes in Math., vol. 41, Springer-Verlag, New York, 1967. 
10. M. Hochster, Big Cohen-Macaulay modules and algebras and embeddability in rings of Witt vectors (Proc. Conf. on Commutative Algebra, Kingston 1975), Queen's Papers in Pure and Appl. Math., vol. 42, Queen's Univ., Kingston, Ontario, 1975, pp. 106-195.

11. M. Hochster and C. Huneke, Tight closure, invariant theory and the Briançon-Skoda theorem, J. Amer. Math. Soc. 3 (1990), 31-116.

12. H. Matsumura, Commutative ring theory, Cambridge Univ. Press, New York, 1986.

13. M. Nagata, Local rings, Interscience, New York, 1962.

14. D. G. Northcott, Finite free resolutions, Cambridge Univ. Press, New York, 1976.

15. T. Ogoma, A note on the syzygy problem, Comm. Algebra 17 (1989), 2061-2066.

16. C. Peskine and L. Szpiro, Dimension projective finie et cohomologie locale, Inst. Hautes Études Sci. Publ. Math. 42 (1973), 323-395.

17. R. Y. Sharp, Cohen-Macaulay properties for balanced big Cohen-Macaulay modules, Math. Proc. Cambridge Philos. Soc. 90 (1981), 229-238.

18. S. Zarzuela, Systems of parameters for non-finitely generated modules and big CohenMacaulay modules, Mathematika 35 (1988), 207-215.

Universität Osnabrück, Standort Vechta, Driverstr. 22, 2848 Vechta, Germany 